\title{
EL DESARROLLO COGNOSCITIVO DE LOS ESTUDIANTES EN EL ÁREA DE LA NATURALEZA CORPUSCULAR DE LA MATERIA
}

\author{
BENARROCH BENARROCH, ALICIA \\ EU de Formación del Profesorado de Melilla. Ctra. Alfonso XIII, s/n. 52005 Melilla. \\ E-mail: aliciabb@goliat.ugr.es
}

\section{SUMMARY}

This paper aims to describe conceptual change with regard to the corpuscular nature of mattter during the development and accumulation of experience. Five levels of explicative schemes have been found, whose construction implie a progressive liberalization of macroscopic features seen in the transformation system. Examples of the different answers in each level are proposed, as well as the most usual changes between levels experimented by the students before and after explaining to them «what do scientist think» about the nature of matter. Finally, conclusions are reached for research on the conceptual change.

\section{INTRODUCCIÓN: LAS INVESTIGACIONES SOBRE, EL DESARROLLO EN DOMINIOS ESPECIFICOS}

Las investigaciones sobre el conocimiento de los estudiantes están experimentando, muy recientemente, cambios significativos que afectan tanto a sus fundamentos teóricos como metodológicos.

En concreto, la línea de investigación sobre concepciones de los alumnos se caracterizaba a mediados de esta década por una falta de progresión (Jiménez Gómez, Solano y Marín, 1997; Marín y Benarroch, 1994) y por una colmatación de resultados de tal índole que incluso ciertos autores llegaron a sugerir un desplazamiento de este centro de interés a otras líneas de investigación (Duschl, 1994; Gil, 1996). Sin embargo, desde nuestro punto de vista, esas críticas sirvieron para inyectar ideas y planteamientos novedosos y para aportar direcciones que prometen impulsarla en lugar de agotarla. Uno de los rumbos adoptados trata de reconciliar la imagen heterogénea que en un momento dado dieron los estudios de concepciones del conocimiento del alumno por otra más homogénea, aunque no tanto como la postulada por Piaget (Pozo et al., 1991). El resultado, aunque heterodoxo, suele estar relacionado en mayor o menor medida con las llamadas teorías de dominio, constituidas por el conjunto de representaciones de diverso tipo activadas por los sujetos ante contextos pertenecientes a un dominio dado (Pozo y Gómez Crespo, 1998), las cuales, a su 
vez, se organizan o se estructuran a partir de una serie de supuestos implícitos, que constituirían una teoría-marco (Vosniadou, 1994) o una teoría implícita (Pozo et al., 1992). Por tanto, se distinguen en la actualidad diferentes grados de generalidad en la organización cognitiva que, al menos a cierto nivel, poseen carácter modular. En nuestro caso, llevamos diez años trabajando con distintos niveles de generalidad cognitiva, a saber, los esquemas operatorios y los específicos (Marín, 1994; Benarroch y Marín, 1997), todos ellos responsables, en último extremo del producto observable de la cognición del sujeto: sus respuestas verbales, escritas, acciones, dibujos, etc.

En este contexto, las investigaciones sobre el desarrollo en dominios específicos prometen ser unas de las más fecundas en didáctica de las ciencias. Su objetivo es entender cómo cambian las estructuras conceptuales en el curso del desarrollo y con la acumulación de experiencia (Vosniadou y Ioannides, 1998). Empieza a recuperarse la conveniencia, si no la evidencia, de que se hace necesaria la investigación sobre la evolución conceptual en dominios específicos y que, por tanto, la resistencia al cambio de las concepciones iniciales de los alumnos podría ser, al menos en ciertos casos, un resultado más aparente que real. Y es que, si aprender ciencia es un proceso gradual durante el cual las estructuras conceptuales iniciales son continuamente enriquecidas y reestructuradas, investigar sobre el aprendizaje implica, al menos, tener intención de conocer:

a) cómo evoluciona con el desarrollo y la experiencia el conocimiento del alumno en un dominio específico;

b) qué mecanismos y barreras cognoscitivas facilitan o dificultan esa evolución;

c) cómo influye en la evolución conceptual «natural» la instrucción específica; y, por último,

d) cómo influyen en la evolución conceptual «natural»e «inducida» las interacciones sociales y otros factores ambientales influyentes en la enseñanza.

Se podría afirmar que la distancia entre las investigaciones con objetivos tipo $a$ y las de objetivos tipo $d$ es la que hay desde la psicología del desarrollo cognoscitivo hasta el dominio propio de la didáctica de las ciencias. También se podría afirmar que la dificultad de hacer planteamientos metodológicos en las investigaciones que controlen rigurosamente los agentes de aprendizaje naturales (derivados de la experiencia natural y del aprendizaje general) e inducidos (derivados de la instrucción específica) es, al tiempo que propia de este tipo de investigaciones sociales, una limitación importante a la hora de interpretar los resultados obtenidos. Teniendo en cuenta esta limitación, y a pesar de ella, las investigaciones sobre el desarrollo cognoscitivo en ámbitos específicos juegan un papel importante y diríamos que imprescindible si lo que se pretende es acoplar la enseñanzaaprendizaje de las ciencias a la evolución cognoscitiva del alumno.

\section{EL CONOCIMIENTO DEL ALUMNO SOBRE LA NATURALEZA CORPUSCULAR DE LA MATERIA}

Si el número de estudios acumulados sobre los conocimientos previos de los alumnos en los últimos veinte años es casi inabarcable (Pfundt y Duit, 1993), uno de los contenidos que más ha contribuido a hacer ingente esta cantidad de publicaciones es el de la naturaleza corpuscular de la materia. Precisamente, uno de los primeros trabajos fue el de Doran (1972), preocupado por obtener buenos distractores en los ítems destinados a valorar la aceptación de la naturaleza atomística de la materia. Furió (1986) ya señaló hace más de una década la abundancia relativa de este tópico respecto a otros en el área de la química. La justificación de la elección de este tópico suele ser muy diversa; en el cuadro I puede verse una relación de algunos de los argumentos o criterios más utilizados.

Esta relación da una rápida imagen de los distintos objetivos con que se afronta la temática de las concepciones en esta área. Este hecho también fue señalado por Eijfelhof y Linjse (1990), en unas reflexiones finales de un seminario que versaba específicamente sobre este tema, quienes lo destacaron como uno de los principales problemas que se plantean al intentar uniformizar la multitud de investigaciones realizadas.

Cuadro I

Algunos argumentos utilizados para justificar las investigaciones en concepciones sobre la naturaleza corpuscular de la materia.

1. Por ser uno de los principales objetivos educativos de la mayor parte de los currículos de ciencias de los cursos superiores de la enseñanza básica (Mitchell y Kellington, 1982; Gabelet et. al., 1987; CLIS, 1987).

2. Por ser de importancia primordial para la ciencia actual y para toda explicación causal de cualquier tipo de cambio material saber que toda la materia está compuesta por partículas y que no es continua (Nussbaum, 1989).

3. Por su poder explicativo y predictivo y, por tanto, por la capacidad de economía mental que supone la utilización de modelos de partículas para explicar cuestiones de la vida cotidiana (Brook et al., 1984).

4. Por su potencialidad para favorecer el trabajo con modelos físicos, acercando la actividad del alumno a la actividad científica (Seré, 1990; Barboux et al., 1987; De Vos, 1990).

5. Para clarificar confusiones de conceptos macroscópicos (energía interna y calor, voltaje y corriente...) (Licht, 1990; Valcárcel et al., 1990).

6. Como requisito indispensable para la iniciación en el ámbito de la química (Llorens, 1987, 1989; Pozo et al., 1991).

7. Para poder desarrollar el mundo conceptual de la biología (Halldén, 1990).

8. Por sus influencias sobre los procesos ecológicos, imprescindibles en la educación ambiental (Helldén, 1995).

9. Por ser un ámbito que ilustra cómo se pueden utilizar los esquemas epistemológicos para seleccionar y secuenciar la instrucción en ciencias (Duschl, 1997), problemas que se plantean a intentar uniformizar la multitud de investigaciones realizadas. 
A pesar de la gran cantidad de investigaciones que tratan de comprender el conocimiento del alumno sobre la naturaleza corpuscular de la materia (Benarroch, 1989; Prieto et al., 1989; Stavy, 1988; Meheut y Chomat, 1990; Sequeira y Leite, 1990; Seré, 1990; Scott, 1992; Gabel, 1993; Benson et al., 1993; Lee et al., 1993; Posada, 1993; 1995; Llorens, 1996; Pozo et al., 1991; Pozo y Gómez Crespo, 1998; etc.), como ya señaló Andersson (1990a, b) hace unos años, una cuestión importante aún sin resolver es el conocimiento de cómo tiene lugar la evolución de las concepciones de los estudiantes sobre la estructura de la materia. Éste ha sido uno de los objetivos principales de un trabajo reciente (Benarroch, 1998a).

El reto importante que se aborda es el de dilucidar cómo evoluciona con la edad y con la experiencia escolar el conocimiento del alumno sobre la naturaleza de la materia. Podría esperarse, de acuerdo con Andersson (1990a), una progresión unidimensional o multidimensional, pero también podría esperarse más persistencia que evolución, asociándose una u otra forma de «ver la materia»a características más personales que evolutivas. Datos a favor de una postura y de otra no faltan: así, para Piaget, esta evolución debía darse de modo «natural» con el desarrollo de las capacidades cognoscitivas. Para los estudiosos de las concepciones alternativas, hay obstáculos epistemológicos que impiden esta evolución, incluso a veces después de la instrucción específica. Los datos experimentales, sin embargo, sugieren que ni la evolución es tan «natural» como Piaget sugiere, ni tan dificultosa como los defensores de las concepciones alternativas han proclamado. Veremos que el marco interpretativo utilizado permite, además de conocer cómo tiene lugar la evolución conceptual, encajar adecuadamente los resultados experimentales de unos y otros y darles una explicación.

\section{METODOLOGÍA}

Aunque el objetivo de este trabajo es presentar los niveles explicativos encontrados en la investigaciones que hemos realizado (Benarroch, 1998a), una breve referencia a la secuencia metodológica resulta imprescindible para valorar el producto obtenido. Es deudora de la teoría piagetiana $\mathrm{y}$, sobre todo, de los últimos trabajos sobre causalidad realizados por el Centro de Epistemología Genética (Piaget y García, 1973). Se fundamenta también en otras vertientes psicológicas que han inspirado el movimiento de las concepciones alternativas, dando importancia especialmente a las investigaciones sobre dominios específicos. No se olvidan las aportaciones teóricas de la epistemología e historia de los modelos de materia. De todo ello y, sobre todo, de la amplia bibliografía que versa sobre el conocimiento del alumno en esta área, se extraen las hipótesis del trabajo.

La metodología se materializa en entrevistas individuales a un número más bien limitado de sujetos (concretamente 43) de distintas edades (9-22 años), pertenecientes a distintos cursos escolares ( $4^{\circ}$ de primaria- universidad) y que tienen distintas capacidades (rendimientos académicos y niveles operatorios variables). Los pasos a seguir fueron:

1) Realización de un estudio piloto $\left(\mathrm{N}_{1}=330\right.$ alumnos $)$ para la selección de la muestra objeto de entrevista individual $\left(\mathrm{N}_{2}=43\right)$ (Benarroch, 1998b).

2) Diseño del conjunto de situaciones físicas. Éstas fueron seleccionadas a partir de un análisis bibliográfico que puso de manifiesto la importancia tanto del estado físico como de los aspectos percibidos sobre la noción corpuscular de la materia. Las situaciones elegidas finalmente fueron: la disolución de un sólido granular, la mezcla del alcohol y del agua y la compresión de un gas. Para cada una de ellas, se plantearon contrapruebas y se modificaron los aspectos figurativos, configurando con ello el conjunto de preguntas fijas que conformaron la entrevista. Véase un ejemplo de tarea en el cuadro II.

3) Realización de las entrevistas individuales.

4) Transcripción de las entrevistas en dos formas:

a) transcripción: literal;

Cuadro II

Fragmento de tarea utilizada en la entrevista.

Comprensión del aire y del agua en una jeringa

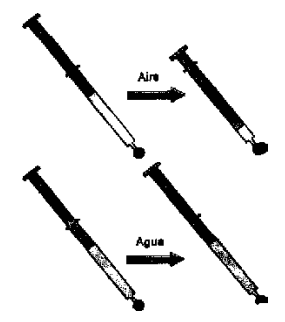

¿Qué diferencia el aire del agua?
La primera parte de esta tarea trata de poner de manifiesto la materialidad de los gases, para lo cual se pesa un globo desinflado y se vuelve a pesar cuando está inflado. A continuación, el alumno «juega» apretando el globo y con una jeringa tapada por su extremo, experimentando la elasticidad del aire. Llegados a este momento, cabe la secuencia de previsión - perturbación - confrontación, que vamos a reproducir.

Fase de previsión ( $G$-ANTES): Se solicitan dibujos y explicaciones sobre la constitución del aire coherentes con su materialidad y su alta compresibilidad. Preguntas implicadas: a) Imagina lo que verías en el interior del globo si tuvieras un microscopio muy potente antes y después de apretarlo. $b$ ) ¿Cómo te imaginas el aire del interior de la jeringa, antes y después de empujar el émbolo, si tuvieras un microscopio muy potente? c) ¿Sería posible reducir totalmente el espacio ocupado por el aire?

Fase de perturbación: Se coloca agua en el interior de la jeringa y se experimenta su incompresibilidad.

Fase de confrontación (G-POSTE): Se solicitan nuevos dibujos y explicaciones sobre la constitución del aire y del agua después de experimentar las distintas compresibilidades. Preguntas implicadas: $a$ ) ¿Qué diferencia el aire del agua? b) ¿Cómo verías el aire y el agua si tuvieras un microscopio muy potente? 
b) transcripción: donde se recogen aspectos más sobresalientes, permitiendo la reducción de los datos obtenidos y haciéndolos más operativos.

5. Categorización de las respuestas de los alumnos y posterior jerarquización (categorías empíricas).

6. Análisis estadístico (especialmente el análisis cluster, factorial y análisis de correspondencias) y recategorización de respuestas (categorías estructurales).

7. Delimitación de los niveles explicativos a merced del contenido evolutivo común en las explicaciones de los sujetos ante las distintas situaciones físicas planteadas.

A continuación, debido al espacio disponible, se expone exclusivamente una de las conclusiones más relevantes de la investigación: los cinco niveles explicativos encontrados sobre la NCM.

\section{LOS NIVELES EXPLICATIVOS DE LOS ALUMNOS SOBRE LA NATURALEZA COR- PUSCULAR DE LA MATERIA}

Aunque una primera impresión después de las entrevistas fuera pesimista en lo que se refiere a alcanzar resultados generales para las respuestas de los alumnos, la categorización de las entrevistas, su tratamiento estadístico y la posterior recategorización permitieron ir progresivamente cambiando este punto de vista por otro cada vez más consensuado. Finalmente, se pudieron alcanzar cinco niveles explicativos en las respuestas de los alumnos. Así:

- El nivel I se caracteriza por una imagen de materia continua y estática, salvo que mayoritariamente se observe lo contrario. Esto es, los alumnos de este nivel son incapaces de trasvasar la barrera de lo observable y, además, no comprenden la necesidad de dar explicaciones a los cambios de la materia. Las cosas ocurren porque sí. Por ejemplo, ante la observación de que el aire es compresible y el agua no, dirá: «Es así. El aire se puede apretar y el agua no». Si el profesor insiste en alcanzar una explicación, llegará a poner ejemplos propios que no pasan de ser una «traducción» de la experiencia con sus propias palabras, pero esa explicación no deja de ser una simple descripción de los observables (decimos que no hay explicación o que ésta se da en el nivel fenomenológico).

- El nivel II se conforma por modelos de materia que siguen siendo continuos, pero que se ven enriquecidos con elementos percibidos (burbujas, huecos, pompitas, etc.) para dar explicación a los datos empíricos. La importancia de los elementos percibidos en este nivel es tal que el modelo de materia se modificará de una sustancia a otra sin prejuicios, con la finalidad de dar explicaciones a los cambios de la materia. Esta necesidad de explicación es lo que realmente caracteriza a este nivel y lo diferencia del anterior. En consecuencia, un alumno puede concebir el agua como un continuo embu- tido de partículas y el aire como un continuo con huecos, intentando explicar las diferencias de compresibilidades. Sus dibujos incluso podrían ser idénticos para ambos, pero ese alumno habría encontrado un «artificio» para dar explicación a los datos empíricos.

- El nivel III constituye el primero de la evolución conceptual que implica concepciones corpusculares. En él, la materia está formada por partículas. Estas partículas son invisibles incluso a nivel microscópico y ya no están, por tanto, relacionadas con la percepción más o menos directa, tal como ocurría en el nivel anterior. Entre las partículas, hay huecos, aunque esos huecos puedan estar indistintamente vacíos (vacuistas) o, como más frecuentemente suele suceder, pueden ser concebidos llenos de «algo» (plenistas). Esto es, lo que diferencia al alumno de este nivel del siguiente es que el de éste no siente la necesidad de que los huecos entre partículas deben estar vacíos, bien porque no diferencia entre vacío y materia (en este caso, hablar de algo, nada, algo como el aire, éter, es prácticamente hablar de lo mismo), o bien porque rechaza epistemológicamente la posibilidad de que haya alguna «zona» del universo sin nada de materia.

Ante los cambios en la materia, se dan explicaciones causales, fundamentadas en:

a)Las partículas hipotéticas, a las que se atribuyen las propiedades percibidas (partículas que se separan o se acercan, que aumentan o disminuyen de tamaño, etc.). De este modo, los cambios macroscópicos de la sustancia estudiada (color, estado físico, aspecto, etc.) son adjudicados a las partículas microscópicas.

b) Los huecos entre partículas, llenos de algún contenido de naturaleza etérea (similar a la «materia subtilis» cartesiana). Así, los cambios macroscópicos son explicados acudiendo a fondos de partículas más o menos compactos, con más o menos huecos, etc.

- En el nivel IV, además de la existencia de partículas en la materia, se considera la del vacío necesario entre las mismas. Esto es, la diferencia entre este nivel y el anterior está en ese vacío, que es rechazado, evitado o ignorado en el nivel anterior, mientras que en este nivel se considera necesario. La materia debe estar formada por huecos y nada más, pues, si hubiera algo, ese algo también tendría que estar formado por partículas y, entonces, ya no sería hueco entre partículas.

Otro aspecto de la imagen de materia de los alumnos de este nivel es que pueden tener o no desarrollado el subesquema de movimiento e interacción entre partículas, pero, en cualquier caso, éste no está coordinado con el de partículas y vacío en un único modelo causal necesario.

El modelo de partículas y vacío a veces resulta suficiente para explicar los cambios de la materia, aunque para ello el alumno acuda a disposiciones con más o menos vacío. Así, por ejemplo, la distinta compresibilidad del aire y del agua podría ser explicada con un modelo para el aire 
con partículas separadas entre sí y otro para el agua con partículas pegadas (y huecos de tipo intersticial). Sin embargo, el alumno de este nivel se caracteriza y se diferencia del anterior en que su modelo de partículas y vacío es mantenido aun cuando le resulte insuficiente para explicar los datos percibidos. Se trata de un modelo necesario, aunque no le resulte suficiente.

- Por último, el nivel $\mathrm{V}$ coincide con el contenido académico de la enseñanza de la naturaleza corpuscular. Por tanto, aquí la materia se concibe como un sistema de interacción entre partículas, moviéndose continuamente, sin nada entre ellas, sólo vacío.

En este último nivel, los subesquemas de movimiento e interacción se coordinan con el de partículas y vacío, que caracteriza el anterior, en un único modelo causal explicativo de los procesos físicos de la materia.

Los niveles anteriores constituyen aproximaciones sucesivas del modelo adoptado por los científicos, demostrando cómo se evoluciona de las concepciones macroscópicas a las microscópicas, pasando por admitir la existencia de objetos hipotéticos no directamente observables a la de huecos entre esos objetos y a la del vacío en dichos huecos. La construcción de estas concepciones implica una liberalización progresiva de las características macroscópicas observadas en el sistema en transformación.
En el cuadro III (Anexo I) puede verse una síntesis de las características principales señaladas anteriormente para cada uno de los niveles. Se distinguen cuatro columnas: la primera y la segunda recogen las características del modelo de materia y del tipo de explicación asociados a cada nivel; en la tercera damos un ejemplo de respuesta típica de cada nivel; por último, en la cuarta se relacionan los alumnos de la investigación junto a sus edades correspondientes.

Es evidente que el hecho de caracterizar a cada alumno por un nivel explicativo tiene unas consecuencias nada despreciables para la investigación en esta área, ya que implica que es mucho más coherente en sus respuestas de lo que una primera impresión de los datos nos parecía sugerir. Por razones de espacio, no podemos explicar cómo debiéramos la metodología utilizada para hacer esta adjudicación. No obstante, sí señalaremos que el nivel explicativo adjudicado a cada alumno procede de la posición que éste ocupa en las categorías estructurales construidas para 12 de las 35 variables elaboradas sobre las respuestas de los alumnos. Sin embargo, hay que advertir que estas 12 variables (identificadas como las variables «fundamentales») surgen de las últimas respuestas obtenidas para el contenido de la naturaleza corpuscular de la materia. Esto nos hace pensar que, probablemente, la estabilidad de los esquemas explicativos encontrados podría estar relacionada con el grado de reflexión implicado en la respuesta; es decir, hay

Cuadro IV

Gráfico de puntuación factorial-edad de los alumnos.

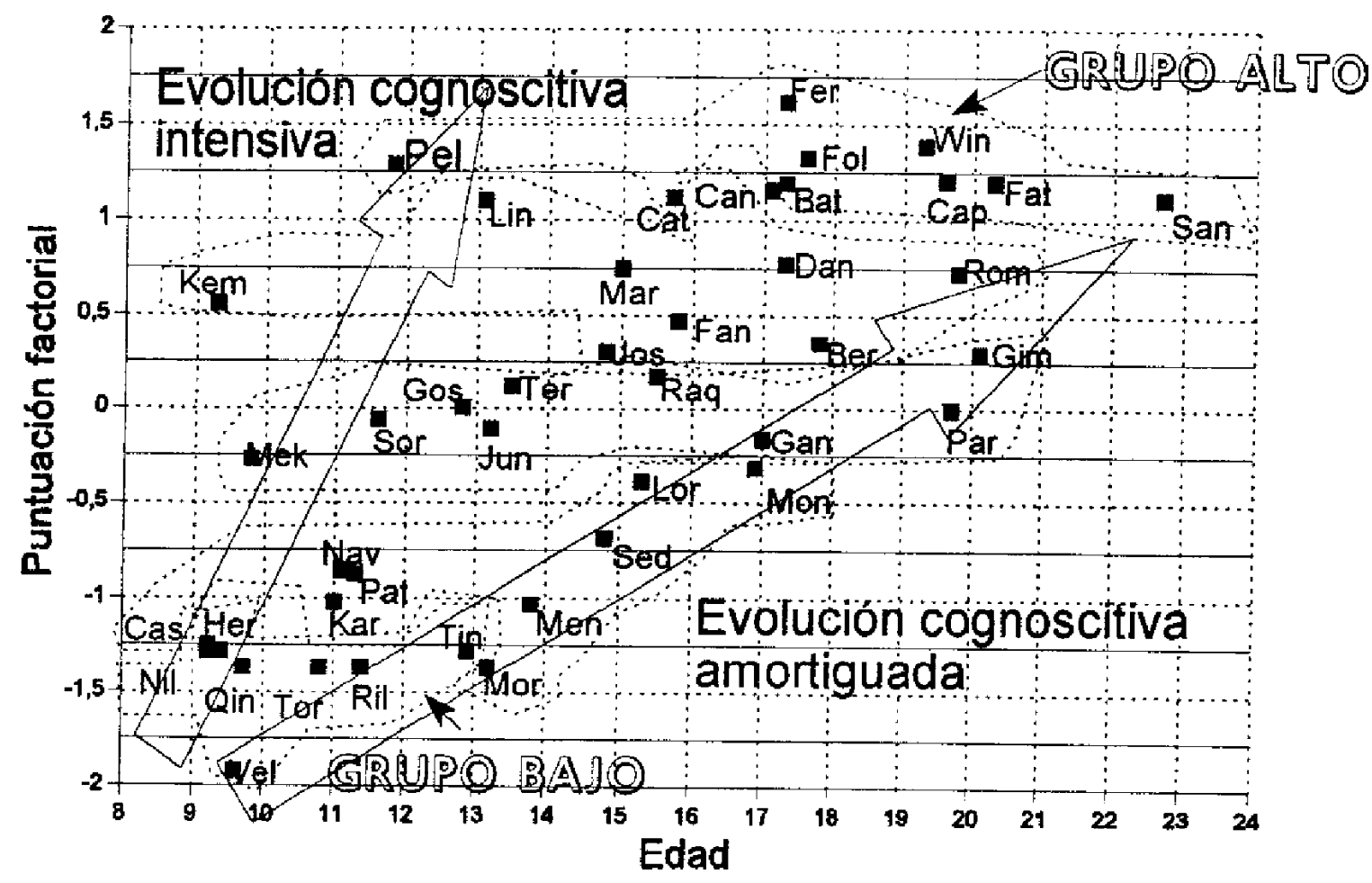


mucha menos estabilidad en las respuestas obtenidas al principio de la entrevista que en las obtenidas al final de la misma, de modo que cuanto mayor sea la oportunidad que se da al alumno para poner a prueba sus primeras ideas (lo que está en función de las contrapruebas diseñadas, del tiempo implicado en la entrevista, etc.), más estabilidad es previsible obtener en sus respuestas. El nivel explicativo vendría a caracterizar al alumno en su estado de «saturación» (situación límite alcanzada en las circunstancias reales de la entrevista).

Otro hecho relevante es que las edades de los alumnos no son buenas predictoras (aunque sí sean sugerentes) de los niveles explicativos encontrados. El gráfico edadpuntuación factorial (magnitud que indica el rendimiento global de los alumnos en la entrevista, obtenida del único factor seleccionado mediante el análisis factorial realizado sobre las 12 variables fundamentales aludidas previamente), que puede verse en el cuadro IV, pone de manifiesto que pueden haber alumnos de 9 años en el nivel IV de la evolución cognoscitiva (Kem), aunque esto no sea lo general (este alumno, así como Pel y Mek habían sido seleccionados por destacar respecto a su grupo-clase; en el gráfico observamos que son sujetos con evoluciones cognoscitivas aceleradas). Asimismo, hay alumnos de 13 años (Tin) que pueden estar en el primer nivel de la evolución cognoscitiva, aunque esto tampoco sea lo más común (sufren una evolución cognoscitiva retardada).

También se desprende del cuadro IV que las edades en que se producen mayores contrastes de niveles entre los estudiantes, al menos para el contenido que nos ocupa, es la de 12-13 años, y que esas diferencias siguen siendo acuciantes hasta los 16-17, lo que parece sugerir una especial dificultad para la enseñanza-aprendizaje de la naturaleza corpuscular de la materia en la educación secundaria.

A continuación, describiremos con detalle las respuestas dadas por los sujetos de la investigación a una de las cuestiones de la entrevista que resultaron más significativas.

\section{RESPUESTAS DE LOS ALUMNOS AGRUPA- DAS POR NIVELES EXPLICATIVOS PARA LA COMPRESIBILIDAD DEL AIRE EN CONTRASTE CON LA DEL AGUA}

Una de las tareas o situaciones físicas que ha resultado ser más fiable para discernir los niveles explicativos de los alumnos sobre la NCM ha sido la experiencia de la compresibilidad del aire en contraste con la del agua (Cuadro II). En ella, después de experimentar con el aire en un globo, comprobar su peso, se experimenta con el de una jeringa, «jugando» con el aire de su interior y comprobando que $« 5 \mathrm{ml}$ se reducen a $1 \mathrm{ml}$. No ocurre lo mismo si, en lugar de aire, se introduce agua (a los ojos de los alumnos, el agua no es compresible). Las preguntas realizadas fueron del siguiente tipo: ¿qué diferencia el aire del agua? ¿Cómo verías el aire y el agua si tuvieras un microscopio muy potente?
Las respuestas dadas por los alumnos (representados con seudónimos) de cada uno de los niveles explicativos fueron las siguientes:

\section{Nivel 1}

- Conciben tanto el aire como el agua continuos. La explicación de las distintas compresibilidades radica en la propia naturaleza de las sustancias. (Qin, Mor, Tor, Ril, Nil, Her, Vel, Tin)

\section{Nivel 2}

- Modelos continuos con huecos solamente para el aire (agua continua) o modelos continuos con huecos para ambas sustancias.

(Lor, Kar, Nav, Sed, Cas)

\section{Nivel 3}

- Modelos «discontinuos aparentes» (partículas/fondo/ lleno) para una de las dos sustancias; la otra sigue siendo continua. La sustancia continua se comporta así por su propia naturaleza. Para la «discontinua aparente», se hace una transposición de la propiedad macroscópica al fondo microscópico.

(Jos, Jun, Mek, Mon, Pat, Gos)

- Modelos «discontinuos aparentes» (partículas y fondo lleno) para ambas sustancias, esto es, se hace una transposición de las propiedades observadas en los fondos microscópicos. Las explicaciones se basan en la naturaleza de esos fondos.

(Raq, Sor, Par, Gan, Dan, Ber, Men, Fan, Gim)

- Modelos «discontinuos rudimentarios» (partículas y vacío) para el aire. El agua sigue siendo «discontinua aparente» (partículas de agua y fondo lleno). Las explicaciones intentan ser algo más elaboradas que la mera transposición al nivel microscópico.

(Ter, Cat, Mar, San, Rom)

\section{Nivel 4}

- Modelos «discontinuos rudimentarios» (partículas y vacío) para ambas sustancias, a pesar de no constituir un sistema lo suficientemente explicativo para dar cuenta de las distintas compresibilidades. Explicaciones bloqueadas.

(Can, Bat, Lin)

- Modelos «discontinuos rudimentarios» (partículas y vacío) para ambas sustancias, introduciendo estrategias o disposiciones (partículas de agua pegadas) que explican las distintas compresibilidades. Explicaciones desbloqueadas.

(Fol, Kem, Fat)

\section{Nivel 5}

- Modelos discontinuos avanzados (partículas/vacío/ fuerzas) para ambas sustancias, lo que se deduce de la 
necesidad de explicar las distintas compresibilidades. (Pel, Win, Cap)

- Modelo académicamente aceptado (partículas/vacío/ fuerzas/movimiento) tanto para el aire como para el agua. (partículas/vacío/fuerzas/movimiento) para ambas sustancias.

(Fer)

Como ya se puede suponer, los niveles alcanzados por los sujetos en esta cuestión no coinciden plenamente (la coincidencia es del 65\%) con los que finalmente se les adjudicaría en la investigación. Esto viene a poner de manifiesto algo que nos parece importante: una sola cuestión, por buena que ésta sea, no puede darnos la misma información cognoscitiva del alumno que el conjunto de las 25 cuestiones extraídas de la entrevista. Y menos aún cuando esas cuestiones se han diseñado con diversas estrategias de variabilidad (de dificultad, de contextos físicos, de variables, etc.) dando cabida a cualquier sistema cognoscitivo.

En la relación anterior, aunque no pueda apreciarse la verdadera amplitud del espectro de respuestas, sí que se pone de manifiesto la aparente similitud entre los dibujos correspondientes a distintos niveles cognoscitivos, siendo posiblemente ésta una de las causas de las contradicciones encontradas en la bibliografía sobre el conocimiento del alumno en esta área. En efecto, formas icónicas aparentemente idénticas representan nociones corpusculares muy diferenciadas y alejadas entre sí. Así, circulitos, puntitos, etc., muy usuales en las representaciones, pueden significar huecos, burbujas similares a las percibidas, algunas partículas seleccionadas del continuo (formado por partículas «más disueltas»), partículas representativas del estado de la materia (por ejemplo, todos los líquidos podrían estar formados por partículas de agua) o partículas representativas de la naturaleza de la sustancia. Las formas icónicas, como significantes que son, evolucionan más lentamente que los significados atribuidos.

\section{CAMBIOS CONCEPTUALES INDUCIDOS POR LA INSTRUCCIÓN}

Hemos mostrado que los esquemas iniciales de los niños sobre la materia sufren una evolución importante con el desarrollo y con el aprendizaje. En principio, no es posible saber cuáles de estos cambios son consecuencia del desarrollo «natural» del niño, de su interacción física y social con su medio, y cuáles son consecuencia de la instrucción específica. Como señalan Vosniadou y Ioannides (1998), algunos cambios podrían no estar directamente relacionados con la enseñanza de las ciencias, mientras que otros sí. Llamaremos a los primeros espontáneos y a los segundos, inducidos o basados en la instrucción.

En nuestro trabajo nos propusimos conocer en qué dirección y cuantía la instrucción sobre el modelo corpuscular de la materia alteraba los esquemas explicativos de los alumnos. Esto es, después de conocer los esquemas «espontáneos», se pretendió indagar en los «inducidos», dadas las repercusiones que esta información podría reportar para la enseñanza. Para ello, se solicitaron nuevas respuestas de los alumnos a las situaciones físicas planteadas en la entrevista después de la explicación de una ficha que contenía algunas de las sentencias claves del modelo corpuscular de la materia (Cuadro V).

Cuadro V

Modelo de ficha (utilizado para la instrucción).

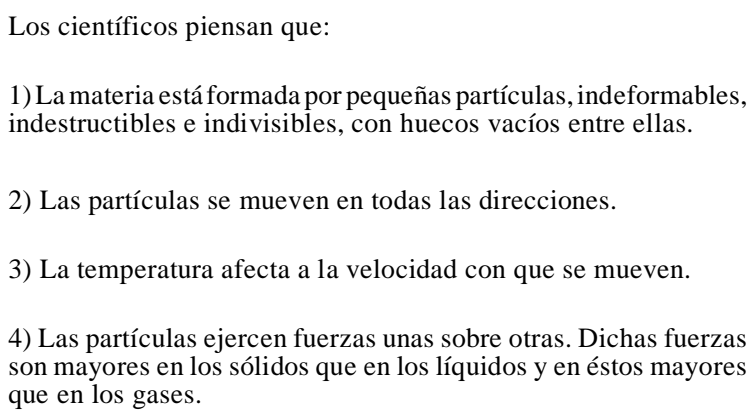

2) Las partículas se mueven en todas las direcciones.

3) La temperatura afecta a la velocidad con que se mueven.

4) Las partículas ejercen fuerzas unas sobre otras. Dichas fuerzas son mayores en los sólidos que en los líquidos y en éstos mayores que en los gases.

El análisis de los esquemas explicativos de los alumnos después de la breve fase de instrucción aportó ciertos datos de sumo interés para la enseñanza de este contenido (Cuadro VI, anexo II):

- El dato más significativo que hay que resaltar es que el orden jerárquico de niveles explicativos se vuelve a encontrar después de la presentación del modelo, lo que indica que los progresos experimentados se inscriben en los niveles de conjunto que ya conocíamos de la evolución natural. Es evidente que alimentando los esquemas del sujeto es posible acelerar su desarrollo cognoscitivo. En el cuadro VI, se pueden apreciar los nuevos niveles alcanzados por los alumnos, en comparación con los «antiguos», para la ya conocida tarea de la compresibilidad del aire y del agua.

- La instrucción de «lo que piensan los científicos sobre la naturaleza de la materia» lleva a los alumnos de cada uno de los niveles a seleccionar la parte de la información presentada para la que tienen esquemas relevantes activados y que además es consistente con el conocimiento presentado por ellos. Así:

- Los alumnos que de forma espontánea son continuos (nivel I) asimilan únicamente el concepto de partícula como significante, sin atribuirle ningún significado microscópico, pues no se detecta enriquecimiento alguno en sus explicaciones. En consecuencia, se limitan a superponer partículas en sus continuos anteriores, tal y como fue señalado por Novick y Nussbaum (1981).

- Los que en la fase espontánea conciben imágenes «continuas con huecos» (nivel II), a veces sólo para el aire y otras tanto para el aire como para el agua, no 
presentan modificaciones en sus concepciones y simplemente rechazan el modelo (permanecen en el nivel II).

- Los sujetos que de forma espontánea conciben partículas sobre fondos continuos para el agua o para el aire, o bien para ambas sustancias (nivel III), pasan a considerar partículas y vacío. Seleccionan, de la información presentada en el modelo, el párrafo que alude a este vacío, por su consistencia con el conocimiento representado en los esquemas previos. En este momento se han encontrado fundamentalmente dos comportamientos diferentes. Por un lado, los alumnos que carecían de herramientas conceptuales para distinguir entre el vacío y la materia, aceptaban el vacío entre partículas, pero sin atribuirle ningún significado. Sus respuestas no experimentaban enriquecimiento, y esto ha sido interpretado como que no sufrieron cambios de nivel explicativo. En cambio, otros alumnos, que sí diferenciaron entre vacío y materia, buscaron en las otras sentencias del modelo la explicación de las distintas compresibilidades, dando respuestas más elaboradas, pertenecientes al nivel IV de esquemas explicativos (cambiaron del nivel III al IV). Por tanto, en términos de cambio conceptual, se detectaron permanencias en el nivel III y cambios en el IV.

- Los sujetos que de forma espontánea son corpusculares y además conciben el vacío necesario sólo para el aire, o tanto para el aire como para el agua, después de explicarles la ficha sobre la materia, encuentran en las interacciones entre partículas una explicación útil para las distintas compresibilidades, bien por primera vez, al eliminar otras causas, o bien por haber sido utilizada previamente. En términos del cambio conceptual, suelen pasar del nivel IV al V.

Los datos anteriores parecen sugerir que los cambios o las progresiones de niveles explicativos más comunes son los que implican el salto de un nivel al siguiente. Eso es lo que ocurre con los sujetos de los niveles I, III y IV, que evolucionan, con la presentación de la ficha, hacia niveles II, IV y V, respectivamente. ¿Por qué, entonces, los sujetos del nivel II no sufren un cambio conceptual análogo y pasan a su correspondiente nivel superior? ¿A qué podría deberse la diferencia de comportamientos de los alumnos de este nivel?

La respuesta a esta pregunta podría sugerir barreras de distinta magnitud en los posibles cambios conceptuales implicados. Concretamente, podría invitar a pensar que hay ciertos cambios plausibles tan sólo con ciertas sugerencias específicamente dirigidas al contenido implicado, a diferencia de otros que requerirían un sustrato cognoscitivo más amplio. Esta interpretación sería coherente con la hipótesis de que las explicaciones de los estudiantes son consecuencia de dos tipos de esquemas cognoscitivos con distinto grado de generalidad; a saber, los esquemas operatorios generales y los esquemas específicos, dependientes del contenido (Benarroch y
Marín, 1997). De este modo, la evolución cognoscitiva entre los dos primeros niveles entre sí, por un lado, y entre los tres últimos, por otro, dependería fundamentalmente del enriquecimiento en esquemas específicos. Sin embargo, el cambio conceptual del nivel II al III, en lugar de tener unos requerimientos específicos podría tenerlos operatorios o, al menos, más generales. Nuestros datos sugieren que este sustrato cognoscitivo no parece que pueda conseguirse al menos en el período de tiempo que duraban las entrevistas.

En consecuencia, creemos que esta discusión podría aportar ciertas ideas y sugerencias a los estudios actuales sobre el cambio conceptual. Por un lado, parece apoyar a los que señalan que las investigaciones sobre el desarrollo en contenidos específicos aportan importantes descripciones de los tipos de cambio conceptual que ocurren durante el proceso de adquisición del conocimiento (Vosniadou y Ioannides, 1998). Por otro, también parece confirmar la importancia de aspectos generales y de aspectos específicos en la cognición del sujeto (Benarroch y Marín, 1997), de manera que los cambios en los primeros tienen mayor coste de esfuerzo, experiencia y tiempo que los cambios de los segundos. Y, por último, al describir la relación estructural entre los conceptos del dominio implicado, sugiere importantes implicaciones para el diseño del currículo y la enseñanza.

\section{CONCLUSIONES}

En este trabajo se han descrito los niveles explicativos de los alumnos cuando se enfrentan a situaciones que pueden ser interpretadas en términos corpusculares. La construcción de estos niveles implica una liberalización progresiva de las características macroscópicas observadas en el sistema en transformación y un acercamiento progresivo de la concepción académica contemplada sobre la naturaleza de la materia y sus cambios. También se ha visto que la instrucción sobre lo que piensan los científicos acerca de la materia favorece los cambios conceptuales de los niveles originales a los contiguos superiores. Esto es cierto en aquellos casos en que la «distancia» entre niveles es de carácter específico. Cuando la barrera que los intercepta es de carácter general, el cambio conceptual resulta más dificultoso y quizás sólo posible a más largo plazo.

Se concluye con la necesidad de impulsar investigaciones sobre el desarrollo cognoscitivo en dominios específicos que, además de analizar cómo tiene lugar éste de modo natural o espontáneo, estudien las relaciones entre las representaciones internas y los procesos que tienen lugar durante la actividad cognitiva con las variables externas y de situación que influyen en ellos. 


\section{REFERENCIAS BIBLIOGRÁFICAS}

ANDERSSON, B. (1990a). Pupil's conceptions of matter and its transformations (age 12-16). Studies in Science Education, 18 , pp. 53-85.

ANDERSSON, B. (1990b). Pupil's conceptions of matter and its transformations (age 12-16), en Linjse, P.L., Licht, P. de Vos, W. y Waarlo, A.J.(eds). Relating Macroscopic Phenomena to Microscopic Particles. A central problem in secondary science education, pp.12-35. Utrech: Universidad de Utrech.

BENARROCH, A. (1989). La naturaleza «particulativa» de la materia. Un estudio longitudinal de ideas previas. Publicaciones, 15, pp. 135-148.

BENARROCH, A.(1998a). «Las explicaciones de los estudiantes sobre las manifestaciones corpusculares de la materia. Descripción, análisis y predicción de características y dificultades.» Tesis doctoral inédita. Universidad de Granada.

BENARROCH, A. (1998b). Un estudio empírico de la relación entre las tareas razonadas de ciencias, el estilo cognitivo DIC y el rendimiento escolar, en Jiménez, M.A. (coord.). Didáctica de las Ciencias y Transversalidad, pp. 407-416. Málaga: Serv. Publ. Univ.

BENARROCH, A. y MARÍN, N. (1997). Dependencia de las explicaciones de los alumnos de esquemas de conocimiento específicos y generales. Comunicación presentada al $V$ Congreso Internacional sobre Investigación en Didáctica de las Ciencias Experimentales. Murcia. Septiembre.

BENSON, D.L., WITTROCK, M.Cy BAUR, M.(1993). Students' preconceptions of the nature of gases. Journal of Research in Science Teaching, 30(6), pp. 587-597.

DORAN, R.L. (1972). Misconceptions on selected concepts held by elementary school students. Journal of Research in Science Teaching, 9(2), pp. 127-137.

DUSCHL, R.A. (1994). Editorial policy statement and introduction. Science Education, 78(3), pp. 203-208.

EIJKELHOF, H.M. y LINJSE, P.L. (1990). Looking back and forward: report of reflections and discussions, en Actas del Seminario «Relating macroscopic phenomena to microscopic Particles. A central problem in Secondary Science School», pp. 328-334. Utrecht: Centre for Studies in Science and Mathematics Education. Universidad de Utrecht.

FURIÓ, C. (1986). Metodologías utilizadas en la detección de dificultades y esquemas conceptuales en la enseñanza de la química. Enseñanza de las Ciencias, 4(1), pp. 73-77.

GABEL, D.L. (1993). Use of the particle nature of matter in developing conceptual understanding. Journal of Chemical Education, 70(3), pp. 193-194.

GIL, D. (1996). New Trends in Science Education. International Journal of Science Education, 18(8), pp. 889-901.

JIMÉNEZ GÓMEZ, E., SOLANO, I. y MARÍN, N. (1997). Estudio de la delimitación de la progresión de las «ideas» del alumno. Enseñanza de las Ciencias, 15(3), pp. 309-328.

LEE, O., EICHINGER, D.C., ANDERSON, C.W., BERKHEIMER, G.D. y BLAKESLEE, T.D. (1993). Changing middle school students'conceptions of matter and molecules. Journal of Research in Science Teaching, 30(3), pp. 247-270.

LLORENS, J.A. (1996). ¿Cómo está constituida la materia? Introducción de un modelo corpuscular a través del desarrollo de situaciones problemáticas. Alambique. Didáctica de las Ciencias Experimentales, 9, pp. 120-130.

MARÍN, N. (1994). Elementos cognoscitivos dependientes del contenido. Revista interuniversitaria de formación del profesorado, 20, pp. 195-208.

MARÍN, N. y BENARROCH, A. (1994). A comparative study of Piagetian and constructivist work on conceptions in science. International Journal of Science Education, 16(1), pp. 1-15.

MEHEUT, M. y CHOMAT.A. (1990). The bounds of children's atomism: an attempt to make children build up a particulate model of matter, en Linjse, P.L. Licht, P. de Vos, W. y Waarlo, A.J. (eds.). Relating Macroscopic Phenomena to Microscopic Particles. A central problem in secondary science education, pp. 266-282. Utrech: Universidad de Utrech.

NOVICK, S. y NUSSBAUM, J. (1981). Brainstorming in the classroom to invent a model: a case study. School Science Review, 62, pp. 771-778.

PFUNDT, H. y DUIT, R. (1993). Bibliography: Students' alternative frameworks and science education. Kiel: Universidad de Kiel.

PIAGET, J. y GARCÍA, R. (1973). Las explicaciones causales. Barcelona: Barral. Traducido de Les explications causales. (1971). París: Presses Universitaires de France.

POSADA, J.M. (1993). Concepciones de los alumnos de 15-18 años sobre la estructura interna de la materia en el estado sólido. Enseñanza de las Ciencias, 11(1), pp. 12-19.

POSADA, J.M.(1995). Contenidos, actividades y/o estrategias de enseñanza de la química en la formación científica de ciudadanos. Encuentro Nacional de Profesores de Matemática, Física, Química y Biología. La Falta. Córdoba. Argentina.

POZO, J.I., PÉREZ ECHEVERRÍA, M.P., SANZ, A. y LIMÓN, M. (1992). Las ideas de los alumnos sobre la ciencia como teorías implícitas. Infancia y Aprendizaje, 57, pp. $3-22$.

POZO, J.I. y GÓMEZ CRESPO, M.A. (1998). Aprender y enseñar ciencia. Madrid: Morata.

POZO, J.I., GÓMEZ CRESPO, M.A., LIMÓN, M. y SANZ, A. (1991). Procesos cognitivos en la comprensión de la ciencia: las ideas de los adolescentes sobre la química. Madrid: Servicio de Publicaciones del MEC.

PRIETO, T., BLANCO, A. y RODRÍGUEZ, A. (1989). The ideas of 11-14 year old students about the nature of solutions. International Journal of Science Education, 11(4), pp. 451-463.

SCOTT, P.H. (1992). Pathways in learning science: A case study of the development of one students's ideas relating to the structure of matter, en Duit, R., Goldberg, F., Niedderes, H. Research in physics learning: Theoretical issues and empirical studies, pp. 203-224. Kiel: IPN.

SEQUEIRA, M. y LEITE, L. (1990). On relating macroscopic phenomena to microscopic particles at the junior high school level, en Linjse, P.L., Licht, P. de Vos, W. y Waarlo, A.J. (eds). Relating Macroscopic Phenomena to Microscopic Particles. A central problem in secondary science education, pp. 220-232. Utrech: Universidad de Utrech. 


\section{INVESTIGACIÓN DIDÁCTICA}

SERÉ, M.G. (1990). Passing from one model to another: with strategy?, en Linjse, P.L., Licht, P., de Vos, W. y Waarlo, A.J.(eds.). Relating Macroscopic Phenomena to Microscopic Particles. A central problem in secondary science education, pp. 50-66. Utrech: Universidad de Utrech.

STAVY, R. (1988). Children's conception of gas. International Journal of Science Education, 10(5), pp. 553-560.
VOSNIADOU, S. (1994). Capturing and Modeling the Process of Conceptual Change. Learning and Instruction, 4(45-6), p. 9.

VOSNIADOU, S. y IOANNIDES, C. (1998). From conceptual development to sicence education: a psychological point of view. International Journal of Science Education, 20(10), pp. 1213-1230.

[Artículo recibido en mayo de 1997 y aceptado en enero de 2000.] 


\begin{tabular}{|c|c|c|c|c|c|c|}
\hline \multirow{5}{*}{ 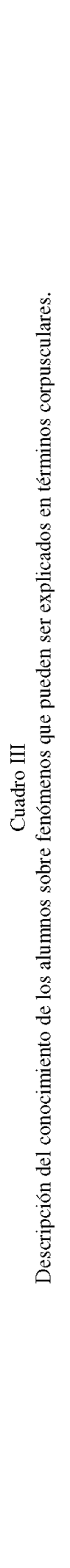 } & 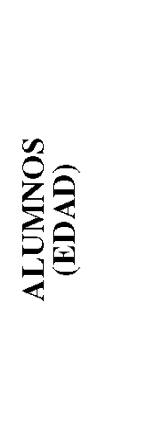 & 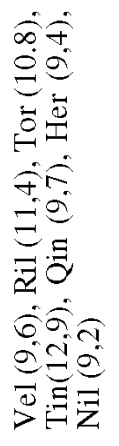 & 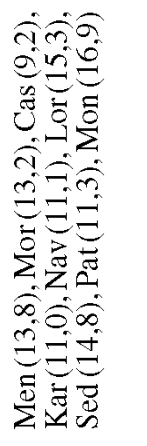 & 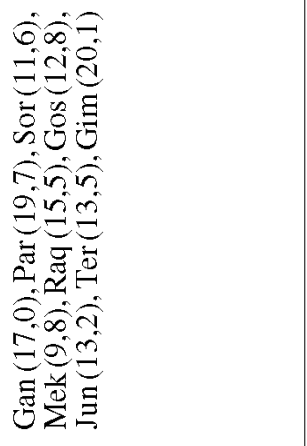 & 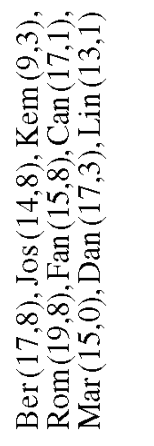 & 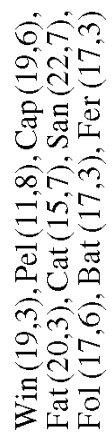 \\
\hline & 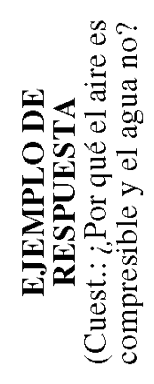 & 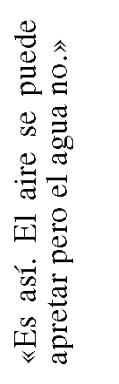 & 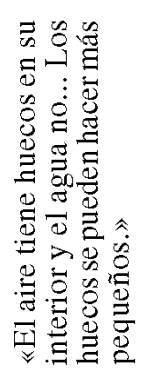 & 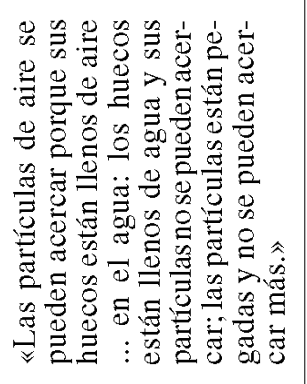 & 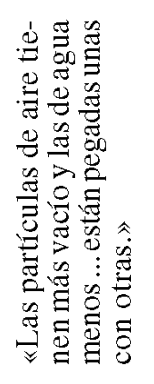 & 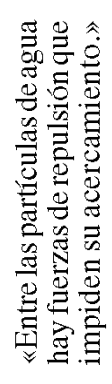 \\
\hline & 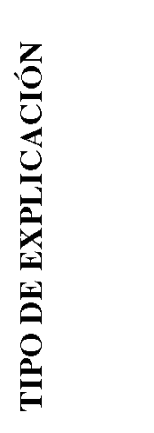 & 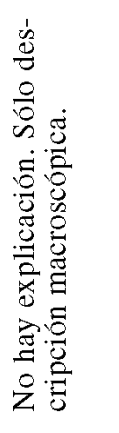 & 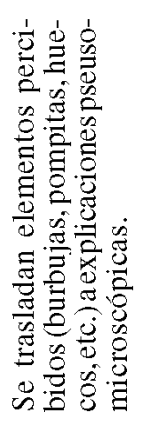 & 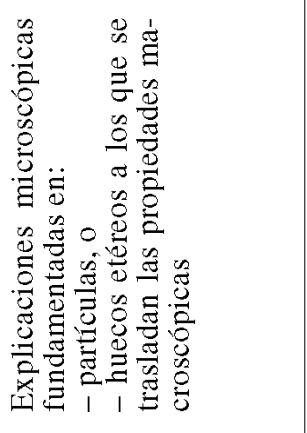 & 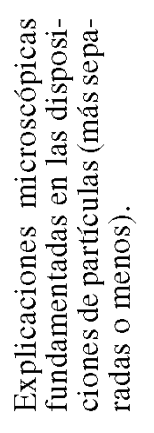 & 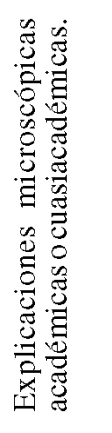 \\
\hline & 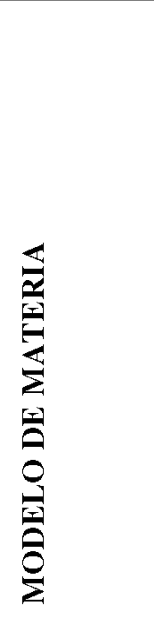 & 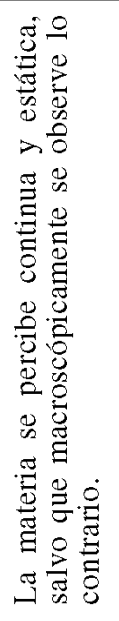 & 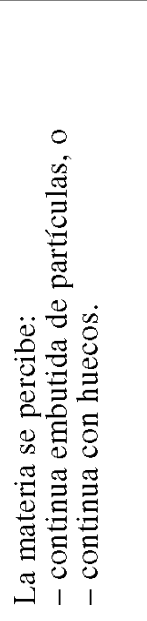 & 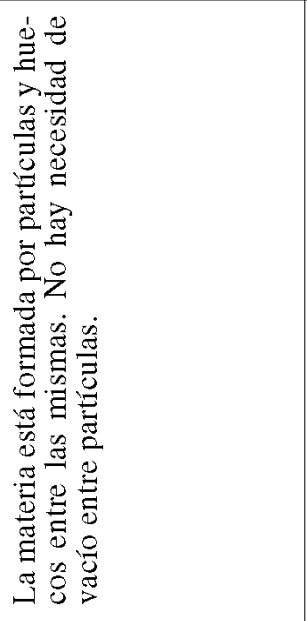 & 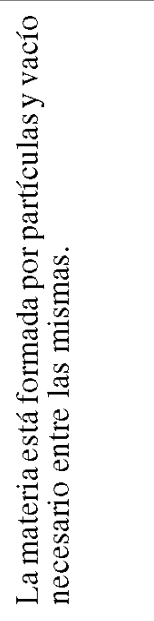 & 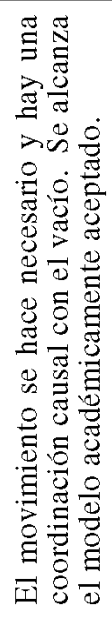 \\
\hline & $\vec{z}$ & $\neg$ & $\Xi$ & $\Xi$ & $\geq$ & $>$ \\
\hline
\end{tabular}




\section{ANEXO 1I}

Cuadro VI

Cambios conceptuales más frecuentes tras la breve instrucción específica.

\begin{tabular}{|c|c|c|c|c|c|c|}
\hline $\begin{array}{c}\text { Nivel } \\
\text { «espontáneo» }\end{array}$ & $\begin{array}{l}\text { Modelo } \\
\text { «espontáneo» }\end{array}$ & Suj. & \multirow{3}{*}{$\Rightarrow$} & Suj. & Modelo «inducido» & $\begin{array}{c}\text { Nivel } \\
\text { «inducido» }\end{array}$ \\
\hline $\mathrm{V}$ & $\begin{array}{l}\text { Partícùlas/vacío } \\
\text { /fuerzas }\end{array}$ & $\begin{array}{l}\text { Fer } \\
\text { Cap } \\
\text { Win } \\
\text { Pel }\end{array}$ & & $\begin{array}{l}\text { Fer } \\
\text { Cap } \\
\text { Win } \\
\text { Pel } \\
\text { Fat }\end{array}$ & \multirow{2}{*}{ Partículas/vacío/fuerzas } & \multirow{2}{*}{$\mathrm{V}$} \\
\hline IV & Partículas/vacío & $\begin{array}{c}\text { Fat } \\
\text { Kem } \\
\text { Fol } \\
\text { Lin } \\
\text { Bat } \\
\text { Can }\end{array}$ & & $\begin{array}{c}\text { Kem } \\
\text { Fol } \\
\text { Lin } \\
\text { Bat } \\
\text { Can }\end{array}$ & & \\
\hline \multirow[t]{3}{*}{ III } & $\begin{array}{l}\text { Aire: Partículas/nada } \\
\text { Agua: Partículas/fondo lleno }\end{array}$ & $\begin{array}{c}\text { Rom } \\
\text { San } \\
\text { Mar } \\
\text { Cat } \\
\text { Lor }\end{array}$ & \multirow[t]{2}{*}{$\Rightarrow$} & $\begin{array}{c}\text { Rom } \\
\text { San } \\
\text { Mar } \\
\text { Cat } \\
\text { Lor }\end{array}$ & Partículas/vacío necesario & IV \\
\hline & $\begin{array}{l}\text { Aire: Partículas/fondo lleno } \\
\text { Agua: Partículas/ fondo lleno }\end{array}$ & $\begin{array}{c}\text { Gim } \\
\text { Fan } \\
\text { Men } \\
\text { Ber } \\
\text { Dan } \\
\text { Gan } \\
\text { Par } \\
\text { Sor } \\
\text { Raq }\end{array}$ & & $\begin{array}{c}\text { Gim } \\
* \\
\text { Men } \\
\text { Ber } \\
* \\
\text { Gan } \\
\text { Par } \\
\text { Sor } \\
\text { Raq }\end{array}$ & $\begin{array}{l}\text { Partículas/vacío } \\
\text { (como significante) }\end{array}$ & \multirow[t]{2}{*}{ III } \\
\hline & $\begin{array}{l}\text { Aire: Partículas/fondo lleno } \\
\text { Agua: Continuo }\end{array}$ & $\begin{array}{c}\text { Jos } \\
\text { Jun } \\
\text { Mek } \\
\text { Mon } \\
\text { Pat } \\
\text { Gos }\end{array}$ & \multirow{2}{*}{$\begin{array}{l}\Rightarrow \\
\Rightarrow \\
\Rightarrow\end{array}$} & $\begin{array}{c}\text { Jos } \\
\text { Jun } \\
\text { Mek } \\
\text { Mon } \\
\text { Pat } \\
\text { Gos }\end{array}$ & & \\
\hline II & Continuos con huecos & $\begin{array}{l}\text { Sed } \\
\text { Cas } \\
\text { Lor } \\
\text { Kar } \\
\text { Nav }\end{array}$ & & $\begin{array}{c}\text { Sed } \\
\text { Cas } \\
* \\
\text { Kar } \\
\text { Nav }\end{array}$ & Continuo con huecos & \multirow[b]{2}{*}{ II } \\
\hline \multirow[t]{2}{*}{ I } & \multirow[t]{2}{*}{ Continuos } & \multirow[t]{2}{*}{$\begin{array}{c}\text { Mor } \\
\text { Nil } \\
\text { Her } \\
\text { Qin } \\
\text { Tin } \\
\text { Tor } \\
\text { Ril } \\
\text { Vel }\end{array}$} & \multirow[t]{2}{*}{$\Rightarrow$} & $\begin{array}{l}\text { Mor } \\
\text { Nil } \\
\text { Her } \\
\text { Qin } \\
\text { Tin } \\
\text { Tor } \\
\text { Ril }\end{array}$ & Continuos con partículas & \\
\hline & & & & Vel & Continuos & I \\
\hline
\end{tabular}

* El comportamiento de estos alumnos es excepcional respecto a la norma establecido. 